\title{
p53 performs an essential role in mediating the oncogenic stimulus triggered by loss of expression of neurofibromatosis type 2 during in vitro tumor progression
}

\author{
XIYE LI $^{1-3^{*}}$, HONGSAI CHEN ${ }^{1-3^{*}}$, LU XUE $^{1-3}$, XIUHONG PANG $^{1-3}$, XIAOMAN ZHANG $^{1-3}$, \\ ZHENGJIE $\mathrm{ZHU}^{1-3}$, WEIDONG ZHU ${ }^{1-3}$, ZHAOYAN WANG ${ }^{1-3}$ and HAO WU ${ }^{1-3}$ \\ ${ }^{1}$ Department of Otolaryngology Head and Neck Surgery, Xinhua Hospital; ${ }^{2}$ Ear Institute, \\ School of Medicine, Shanghai Jiao Tong University, Shanghai 200092; \\ ${ }^{3}$ Shanghai Key Laboratory of Translational Medicine on Ear and Nose Diseases, Shanghai 200092, P.R. China
}

Received September 15, 2015; Accepted March 10, 2017

DOI: $10.3892 / \mathrm{ol} .2017 .6445$

\begin{abstract}
The loss of the tumor suppressor neurofibromatosis type 2 gene, encoding merlin, has been considered to be a fundamental event during the malignant progression of various cell types. However, a consensus for the mainstream mechanism, by which merlin deficiency contributes to uncontrolled cellular proliferation, has not been reached. The present study aimed to determine whether silencing of merlin using lentivirus-based short hairpin RNA potentiates cellular proliferation and cell cycle progression in human colon carcinoma HCT116 cell lines, expressing p53. The present results demonstrated that merlin knockdown contributed to cellular proliferation and G1/S cell cycle progression to a greater extent in HCT116 cells wide-type for p53 (p53 $\left.{ }^{\mathrm{wt}}\right)$ compared with p53-null $\left(\mathrm{p} 53^{-/}\right)$cells. This was supported by overexpression experiments which demonstrated a significant inhibitory effect of excess merlin on cellular proliferation only in HCT116 $553^{\text {wt }}$ cells. In order to investigate the underlying mechanisms of action, the expression of p53-involved G1/S transition genes was evaluated by western blot analysis. For HCT116 p53 ${ }^{\mathrm{wt}}$ cells, merlin loss suppressed p53 expression, and therefore the dysregulation of cell cycle regulatory proteins, including p21, cyclin D1/cyclin-dependent kinase (CDK) 4 and cyclin E1/CDK2 complexes. However, merlin knockdowns had no impact on the expression of any of the
\end{abstract}

Correspondence to: Dr Zhaoyan Wang or Dr Hao Wu, Department of Otolaryngology Head and Neck Surgery, Xinhua Hospital, School of Medicine, Shanghai Jiao Tong University, 1665 Kongjiang Road, Shanghai 200092, P.R. China

E-mail:wzyent@126.com

E-mail:wuhao622@sina.cn

\section{${ }^{*}$ Contributed equally}

Key words: neurofibromatosis type 2 gene, merlin, p53, cellular proliferation, cell cycle aforementioned molecules in $\mathrm{p} 53^{-/-}$cells, indicating that lack of merlin resulted in G1/S cell cycle progression, and thereby uncontrolled cellular proliferation mainly via the regulation of p53-mediated pathways. Taken together, it was proposed that $\mathrm{p} 53$ performs an essential role in mediating the oncogenic stimulus triggered by merlin loss, and p53 is a molecule that should be investigated for its potential in targeted drug therapy for merlin-deficient malignancies.

\section{Introduction}

The neurofibromatosis type 2 (NF2) tumor suppressor gene was first known for its association with neurofibromatosis type 2 , a hereditary neoplasia syndrome characterized by the occurrence of multiple intracranial tumors, including schwannomas, meningiomas and ependymomas (1-4). Since then, NF2 mutations have also been reported in colorectal (5) and thyroid cancer (6), melanoma (7) and mesotheliomas (8), indicating a more general tumor suppressive role of $N F 2$ in various cell types. The human NF2 gene comprises 17 exons, which encode a 595-amino acid protein termed merlin (9). Merlin protein exhibits high sequence homology to the membrane-cytoskeleton associated ezrin/radixin/moesin family and is implicated in the regulation of several fundamental biological processes, including contact-dependent inhibition of proliferation, cell-cell communication and cell-matrix interactions, all of which are important for tumor initiation and progression (10).

Significant differences in types of $N F 2$ gene mutations have been demonstrated in neurofibromatosis type 2, sporadic schwannomas and other tumor types (11). Nonsense and frameshift mutations are expected to cause truncated gene products, leading to loss of merlin expression from the mutated NF2 allele, whilst merlin harbouring missense mutations are supposedly stably expressed but exhibit increased degradation activity $(12,13)$. Lack of functional merlin has been hypothesized to trigger the dysregulation of a wide variety of signaling cascades from the cell surface to the nucleus, including (but not limited to) growth factor receptors, Ras/Rac downstream effectors, phosphoinositide 3-kinase/protein kinase B and cullin 4-RING ubiquitin ligase DDB1 and CUL4-X-box (14-17). 
However, a consensus for the mainstream mechanism, by which merlin loss contributes to uncontrolled proliferation or dysregulation of the cell cycle pattern in mutation-bearing cells during tumor formation/progression, has not yet been reached.

An ever-increasing breadth of evidence has proposed that tumor protein p53 (TP53), a classical tumor suppressor gene, is associated with $N F 2$ in several genetic and protein studies (18-21). Loss of functional NF2-TP53-double mutant mice exhibited an increased predisposal to neoplasms compared with each of the single mutant mice (18). In a cohort of patients with sporadic meningioma (19), loss of heterozygosity of NF2 coupled with TP53 polymorphism increased the risk of tumor progression. A positive association between the two tumor suppressor products has been identified in glioma cell lines and meningiothelial meningiomas (20). Furthermore, merlin and p53 downstream p21 were revealed to regulate each other in Schwann cells and schwannomas (21). However, little is currently known regarding the contribution of p53 to the decreased tumor suppression in the absence of merlin. This appears to be important for the clarification of mechanisms responsible for merlin-deficient pathogenesis, and particularly the development in potential areas to augment current modalities of treatment.

A more recent study (7) has shown that merlin loss caused by NF 2 mutations in sporadic colorectal carcinomas was associated with an advanced tumor-stage, establishing the role of merlin as a tumor suppressor in colorectal cancer progression. The present study aimed to elucidate the exact role of p53 in the merlin regulation of cellular proliferation, using human colon carcinoma HCT116 cell lines expressing p53 or not expressing p53.

\section{Materials and methods}

Cell lines and cell culture. The HCT116 wild-type for p53 $\left(\mathrm{p} 53^{\mathrm{wt}}\right)$ and HCT116 p53-null (HCT116/379.2, p53 ${ }^{-/}$) cell lines were purchased from the Shanghai Institute of Cell Biology, Chinese Academic of Science (Shanghai, China). The two cell types were grown in McCoy's 5A medium (Invitrogen; Thermo Fisher Scientific, Inc., Waltham, MA, USA) supplemented with $10 \%$ fetal bovine serum and $1 \%$ penicillin/streptomycin at $37^{\circ} \mathrm{C}$ in a humidified incubator with $5 \% \mathrm{CO}_{2}$.

NF2/merlin overexpression and 5-ethynyl-20-deoxyuridine $(E d U)$ incorporation assay. For merlin overexpression experiments, the recombinant pcDNA-NF2 or empty plasmid vector control (ViGene Biosciences, Inc., Rockville, MD, USA) were transfected into indicated HCT116 cell lines using Lipofectamine 2000 reagent (Invitrogen; Thermo Fisher Scientific, Inc), according to the manufacturer's protocol. On day 2 after transfection, cultures in each of the experiment groups and control groups were examined for the expression of NF2/merlin. EdU (Guangzhou RiboBio Co., Ltd., Guangzhou, China) labeling was used to investigate the effect of merlin overexpression on DNA synthesis, which is an indicator of cellular proliferation. Briefly, indicated cells were seeded onto 96-well plates at $8 \times 10^{3}$ cells/well, transfected with $N F 2$-expressing or empty vector at $37^{\circ} \mathrm{C}$ for $48 \mathrm{~h}$, and then exposed to $50 \mu \mathrm{l} \mathrm{EdU}$ for an additional $24 \mathrm{~h}$ at $37^{\circ} \mathrm{C}$. The cells of each well were then washed with PBS, fixed with $4 \%$ formaldehyde at room temperature for $20 \mathrm{~min}$ and incubated with $0.3 \%$ Triton X-100 for $20 \mathrm{~min}$ at room temperature. Subsequently, cultures were reacted with $80 \mu \mathrm{l}$ of $1 \mathrm{X}$ Apollo ${ }^{\circledR}$ reaction cocktail (Guangzhou RiboBio Co., Ltd.) at room temperature for $20 \mathrm{~min}$. The DNA contents of cells in each well were stained with $10 \mu \mathrm{g} / \mathrm{ml}$ DAPI (Beyotime Institute of Biotechnology, Haimen, China) at room temperature for $15 \mathrm{~min}$. Images were captured under LAS AF software V4.3 (Leica Microsystems, Inc., Buffalo Grove, IL, USA). In total 3 fields of view were randomly selected from each analysis; EdU-positive cells were identified from the fluorescent images. Cells were counted with ImageJ v1.43 software (National Institutes of Health, Bethesda, MD, USA). Quantification of EdU incorporating-cells was calculated as follows: EdU-positive cell numbers (red dots)/total numbers (blue dots) $x 100 \%$. A total of $\geq 3$ random images were captured from each well and counted by 3 individuals. Experiments were repeated 3 times.

NF2/merlin-knockdown. The pGC-FU-green fluorescence protein (GFP) lentivirus vector, containing an independent open reading frame for GFP, was used to produce small interfering RNA molecules that inhibit expression of target genes of infected cells. Short hairpin RNAs (shRNAs) were synthesized, annealed and inserted into the lentivirus vector. Briefly, sense and antisense primer containing the sense siRNA (small interfering RNA) sequence, 9 bp loop sequence, antisense siRNA sequence and RNA polymerase III terminator sequence were created with AgeI and EcoRI restriction sites on the 5' and $3^{\prime}$ ends, respectively. Subsequently pGCsiL-U6 (GeneChem Co., Ltd., Shanghai, China) was linearized using by digestion with AgeI and EcoRI, these primers were annealed and inserted into pGCsiL-U6 downstream of the U6 RNA polymerase III promoter following the manufacturer's protocol. In order to obtain optimized conditions, four candidate shRNAs (sh) targeting different NF2 gene coding sequences (Table I) were designed using the siDESIGN Center tool (http://dharmacon. gelifesciences.com) and synthesized by GeneChem Co., Ltd.. In preliminary experiments, two shRNAs (sh1 and sh2) were revealed to exhibit ideal merlin knockdown effects (data not shown) and were therefore utilized in the subsequent investigations. A nonsense shRNA was also constructed as control using the target sequence 5'-TTCTCCGAACGTGTCACG T-3' (GeneChem Co., Ltd.). Two lentivirus-mediated shRNAs against NF2 (Lv-NF2-shRNAs) and a control shRNA (Lv-Con-shRNA) were used to transfect HCT116 cell lines at a multiplicity of infection of 10 . To screen the target for the most effective type of viral transfection, the percentage of GFP-positive cells in total cell numbers was evaluated under the fluorescence microscope (x100 magnification; $\geq 4$ fields of view) 3 days after transduction.

Immunofluorescence of merlin protein. Briefly, cells were seeded onto 24-well plates with a density of $1 \times 10^{4}$ cells/well, cultured at $37^{\circ} \mathrm{C}$ for $48 \mathrm{~h}$, fixed with $4 \%$ formaldehyde at room temperature for $30 \mathrm{~min}$, washed 3 times with PBS and then permeabilized with $0.3 \%$ Triton $\mathrm{X}-100$ at room temperature for $20 \mathrm{~min}$. Following incubation in blocking buffer (0.05\% BSA; Beyotime Institute of Biotechnology) at room temperature 
for $1 \mathrm{~h}$, coverslips were incubated with a rabbit polyclonal anti-merlin antibody (dilution, 1:400; cat. no. HPA003097; Sigma-Aldrich; Merck KGaA, Darmstadt, Germany) at $37^{\circ} \mathrm{C}$ for $1 \mathrm{~h}$, incubated at $37^{\circ} \mathrm{C}$ for $1 \mathrm{~h}$ with red fluorescence-tagged goat anti-rabbit secondary antibody (dilution, 1:400; cat. no. SAB4600407; Sigma-Aldrich; Merck KGaA), and then counterstained with DAPI.

Cellular proliferation and colony formation. The effect of merlin knockdown on cellular proliferation was determined by Cell Counting Kit-8 (CCK-8) from Dojindo Molecular Technologies, Inc. (Kumamoto, Japan). Briefly, cells were seeded onto 96 -well plates $\left(4 \times 10^{3}\right.$ cells/well) and grown at $37^{\circ} \mathrm{C}$ for $0,24,48$ or $72 \mathrm{~h}$. At each specified time point, $10 \mu \mathrm{l} /$ well CCK-8 solutions were added and cells were incubated for an additional $4 \mathrm{~h}$ at $37^{\circ} \mathrm{C}$. Absorbance of wells was measured using a SpectraMax190 microplate reader (Molecular Devices, LCC, Sunnyvale, CA, USA), and absorbance values (optical density) obtained from three aliquots were averaged to produce a single value representing cell viability. The absorbance was then expressed in numerical values, which were finally subjected to statistical analysis. Colony formation experiments were also performed to confirm the effect of merlin knockdown on cellular proliferation. Briefly, cells were seeded onto $6-\mathrm{cm}$ dishes at a density of 500 cells per dish and cultured at $37^{\circ} \mathrm{C}$ for 1 week. At the end of the incubation period, numbers of visible cell colonies were estimated with Wright Giemsa staining (Beyotime Institute of Biotechnology).

Cell cycle analysis. Propidium iodide (PI) staining was conducted to examine the effect of merlin knockdown on cell-cycle distributions. Briefly, $8 \times 10^{5}$ cells were seeded onto $6-\mathrm{cm}$ dishes at $37^{\circ} \mathrm{C}$ for $18 \mathrm{~h}$ prior to analysis. Cells were then trypsinized, washed and fixed in $1 \mathrm{ml} 70 \%$ ethanol at $4^{\circ} \mathrm{C}$ overnight, followed by centrifugation at $1,811 \mathrm{x} \mathrm{g}$ for $5 \mathrm{~min}$ at room temperature, incubation with $100 \mu \mathrm{g} / \mathrm{ml}$ RNase and staining with $50 \mu \mathrm{g} / \mathrm{ml}$ PI for $15 \mathrm{~min}$ in the dark at room temperature. The DNA content of cells was analyzed using a Cell Lab Quanta ${ }^{\mathrm{TM}}$ SC flow cytometer (Beckman Coulter, Inc., Brea, CA, USA).

Protein immunoblotting analysis. The harvested cells were lysed with radioimmunoprecipitation assay buffer (Beyotime Institute of Biotechnology) and centrifuged at 16,060 x g for $15 \mathrm{~min}$ at $4^{\circ} \mathrm{C}$. Subsequently, the supernatant was removed and total cellular protein concentration was assessed using a Bio-Rad protein assay kit (Beyotime Institute of Biotechnology) according to the manufacturer's protocol. Protein samples were separated by $8 \%$ SDS-PAGE and transferred to polyvinylidene fluoride membranes (EMD Millipore, Billerica, MA, USA). The membranes were then separately incubated with primary antibodies as follows: Mouse anti-human $\beta$-actin monoclony antibody (dilution, 1:2,000; cat. no. A5441) was obtained from Sigma-Aldrich (Merck KGaA); rabbit anti-human merlin (dilution, 1:1,000; cat. no. sc-331), mouse anti-human p53 (dilution, 1:1,000; cat. no. sc-126), rabbit anti-human p21 (dilution, 1:1,000; cat. no. sc-397), rabbit anti-human cyclin D1 (dilution, 1:1,000; cat. no. sc-753), rabbit anti-human cyclin E1 (dilution, 1:1,000; cat. no. sc-481), rabbit anti-human CDK2 (dilution,
Table I. Target sequences of four candidate shRNAs.

\begin{tabular}{|c|c|}
\hline shRNA & Target sequences \\
\hline $\operatorname{shRNA} 1^{\mathrm{a}}$ & 5'-GGAAGCAACCCAAGACGTTCA-3' \\
\hline $\operatorname{shRNA2}{ }^{a}$ & 5'-GCTCTGGATATTCTGCACAAT-3' \\
\hline shRNA3 & 5'-ACTTCAAAGATACTGACAT-3' \\
\hline shRNA4 & 5'-TTCGTGTTAATAAGCTGAT-3' \\
\hline
\end{tabular}

${ }^{a}$ Used in formal experiments. shRNA, short hairpin RNA.

1:1,000; cat. no. sc-163) and rabbit anti-human CDK4 (dilution, 1:1,000; cat. no. sc-260) polyclonal antibodies were purchased from Santa Cruz Biotechnology, Inc. (Dallas, TX, USA). Primary antibody binding was detected by horseradish peroxidase-conjugated goat anti-mouse (dilution, 1:2,000; cat. no. A0216; Beyotime Institute of Biotechnology) and anti-rabbit (dilution, 1:2,000; cat. no. A0208; Beyotime Institute of Biotechnology) secondary antibodies, and visualized using the ECL plus system (GE Healthcare Life Sciences, Chalfont, UK).

Reverse transcription-polymerase chain reaction (RT-PCR) analysis. RNA extraction from $1 \times 10^{6}$ cells was performed using TRIzol reagent (Takara Bio, Inc.), according to standard methodology (22), and stored at $-80^{\circ} \mathrm{C}$. The yield and quality of extracted RNA was determined using a NanoDrop 2000 spectrophotometer (Thermo Fisher Scientific, Inc.). All RNA samples were quantified spectrophotometrically at 260 and $280 \mathrm{~nm}$. High quality RNA with A260/A280 ratios of $\sim 2.0$ was used to produce cDNA by reverse transcription by PrimerScript $^{\mathrm{TM}}$ RT reagent kit (Takara Bio, Inc.) according to the manufacturer's protocol. PCR amplification was performed using SYBR ${ }^{\circledR}$ Premix Ex Taq ${ }^{\mathrm{TM}}$ (Takara Bio, Inc.). The PCR protocol was as follows: $95^{\circ} \mathrm{C}$ for $30 \mathrm{sec}$, followed by 40 cycles of $95^{\circ} \mathrm{C}$ for $5 \mathrm{sec}$ and $60^{\circ} \mathrm{C}$ for $34 \mathrm{sec}$, with a final dissociation stage. Amplification and quantitative PCR measurements were performed using the CFX96 RT-PCR Detection System (Bio-Rad Laboratories, Inc.). The relative expression of the human NF2 gene was normalized to $\beta$-actin as endogenous housekeeping gene, and values were obtained by the comparative threshold cycle method $\left(2^{-\Delta \Delta C q}\right)(22)$. The PCR primer sequences were constructed by Sangon Biotech Co., Ltd. (Shanghai, China) and listed as follows: NF2 forward, 5'-ACC GTTGCCTCCTGACATAC-3' and reverse, 5'-TTCAAGGCC TCGATTTCTGT-3'; $\beta$-actin forward, 5'-ACCGAGCGCGGC TACAG-3' and reverse, 5'-CTTAATGTCACGCACGATTTC C-3'. Experiments were repeated 3 times with similar results.

Statistical analysis. Unless otherwise stated, all data are presented as the mean \pm standard deviation from at least three independent experiments. Student's t-test and one-way ANOVA were used to determine differences between groups. $\mathrm{P}<0.05$ was considered to indicate a statistically significant difference. All statistical analyses were conducted using MS Excel software 2013 (Microsoft Corporation, Redmond, WA, USA) and SPSS v17.0 statistics software (SPSS, Inc., Chicago, IL, USA). 


\section{Results}

Anti-proliferative effects of NF2/merlin expression are affected by $p 53$ status in HCT116 cells. Previous studies have reported that the expression of wild-type merlin in normal Schwann cells, schwannoma and mesothelioma cells can inhibit cellular proliferation and arrest cell growth at the G0/G1 phase $(21,23,24)$. The present study first attempted to investigate whether 553 performs a role in modulating the cellular proliferation suppressive threshold to excess merlin. Wild-type and p53-null HCT116 cell lines were transfected with the NF2-expressing vectors (empty vectors as control) under the same condition, and exhibited elevated transcription levels of NF2 2 days after transfection, as described by RT-PCR analysis. Additionally, merlin protein levels were increased $>2$-fold, to a similar degree in the two cell types (Fig. 1A and B). Considering the short duration of overexpression effect in the transient transfection model, EdU staining was conducted to detect the newly synthesized DNA, thereby determining the proliferation activities. As is demonstrated in Fig. $1 \mathrm{C}$ and D, the percentage of EdU-positive cells in the $\mathrm{S}$ phase of the cell cycle was significantly lower in HCT116 p53 $3^{\mathrm{wt}}$ cells with the NF2-expressing vector compared with those with empty vector control $(18.8 \pm 2.5$ vs. $2.4 \pm 1.7 \%$; $\mathrm{P}<0.001)$. However, transfection of the NF2-expressing vector did not confer significant growth inhibition in HCT116 $\mathrm{p} 53^{-/}$cells compared with the control $(23.9 \pm 3.8$ vs. $21.6 \pm 2.7 \% ; \mathrm{P}=0.238)$. These results indicated that p53 may perform an essential role in mediating the inhibitory effect of merlin expression on cellular proliferation.

Enhancement of cellular proliferation is induced by merlin depletion in a p53-dependent manner. The loss of merlin is one of a number of molecular changes that occur in tumor suppressor genes during the malignant progression of colorectal cancer (7). For HCT116 cell lines, no detectable NF2 mutations were observed through direct PCR sequencing of genome DNA (data not shown), indicating that they may possess a wild-type active form of merlin. Knockdown of merlin was then performed using lentivirus-mediated shRNA transfection into HCT116 cultures (wild-type and p53-null), to mimic tumor progression in vitro.

To superficially evaluate the transduction efficacies of lentiviral vectors and the degree of merlin knockdown on HCT116 cultures, fluorescence of lentiviral GFP and merlin protein was observed under the microscope, comparing NF2 shRNA-transfected cultures (experiment group) with non-transfected cultures (blank control) and control shRNA-transfected cultures (negative control). As id demonstrated in Fig. 2, lentiviral vectors in the each of experiment groups and control groups had $>80 \%$ transduction efficiencies, and fluorescence degree of merlin was decreased by $>50 \%$ in experiment groups compared with the controls. Similar results were achieved from HCT116 $\mathrm{p} 53^{-/}$cells under the same transfection conditions (data not shown).

The extent of reduction in merlin expression was then assessed in each of the experimental groups in the indicated HCT116 cell lines compared with negative controls, and all experimental groups demonstrated $>50 \%$ reduction in mRNA level and $>80 \%$ reduction in protein level (Fig. 3A and B). Since uncontrolled proliferation is one of the characteristics of malignancies (6-7), the effect of merlin knockdown on cellular proliferation in HCT116 cell lines, expressing p53 or not, was then examined. In line with observations from merlin overexpression experiments, the CCK- 8 assay demonstrated that merlin-knockdown significantly enhanced cellular proliferation rates in HCT116 p53 ${ }^{\mathrm{wt}}$ cells, but not $\mathrm{p} 53^{-/-}$cells 2 days after seeding (Fig. 3C and D). Notably, the aforementioned results were confirmed by the colony formation assay, indicating that the numbers of colonies were evidently increased only in merlin-knockdown p5 $3^{\mathrm{wt}}$ cells. This finding supports the hypothesis that merlin deficiency triggers uncontrolled cellular proliferation in a p53-dependent manner.

Knockdown of merlin abrogates p53-invovled G0/G1 checkpoint, thus promoting cell cycle progression. Merlin and p53 tumor suppressor products share overlapping roles in regulating the G0/G1 checkpoint and thereby controlling G1/S transition $(21,25,26)$. The presence of uncontrolled proliferation in wild-type HCT116 triggered by merlin depletion may be attributed to the dysregulation of cell cycle progression. Based on these points, it was then investigated whether merlin knockdown affected cell cycle pattern in HCT116 cell lines and, if so, whether this was affected by p53 status.

On day 3 post-transfection of lentiviral shRNAs, indicated HCT116 cell lines in each of the experiment groups and control groups were harvested and divided into two parts: One part was subjected to western blotting aiming to verify the presence of efficient merlin silencing and if successful (data not shown), the remaining part was subjected to the analysis for cell cycle pattern. It was identified that merlin-targeted shRNAs (sh1 and sh2) conferred significant decreases in the percentage of cells in the G0/G1 phase and increases in the percentage of cells in the S phase in HCT116 p5 $3^{\mathrm{wt}}$ cells, but not in HCT116 $\mathrm{p} 53^{-/}$cells, as compared with their control groups (Fig. 4). Considering the critical role of p53 in regulating the G0/G1 checkpoint, these observations indicated that loss of merlin may abrogate p53-dependent G0/G1 checkpoint, thereby contributing to the transition of cell cycle between the G1 and S phase, which is critical for cellular proliferation.

Cell cycle progression by NF2 silencing involves $p 53$ regulation of G1/S transition genes. To investigate the molecular mechanisms by which merlin knockdown contributes to cell cycle progression, the expression of molecules involved in the G1/S transition of the cell cycle was evaluated. It has been widely accepted that G1- to S-phase progression is tightly regulated by $\mathrm{p} 53$ downstream $\mathrm{p} 21$ protein in association with cyclin-dependent kinases (CDKs 2, 4, 6) and their essential activating coenzymes (cyclins D and E) $(1,21,26,27)$. As is demonstrated in Fig. 5, the downregulation of p53-p21 cascade signaling, followed by the upregulation of cyclin D1/CDK4 and cyclin E1/CDK2 complexes were observed in the HCT116 p53 $^{\mathrm{wt}}$ cell line compared with the control. This was comparable to observations in our previous study (21), in which merlin-deficient schwannomas exhibited reduced p21 protein levels and heightened expression of cyclin D1/CDK4 and cyclin E1/CDK2 complexes. As expected, no 
A

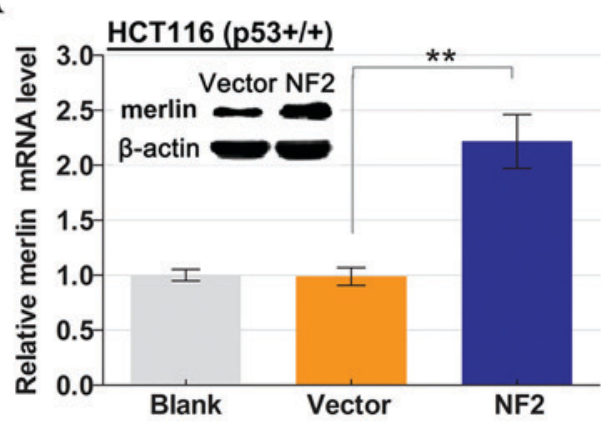

C
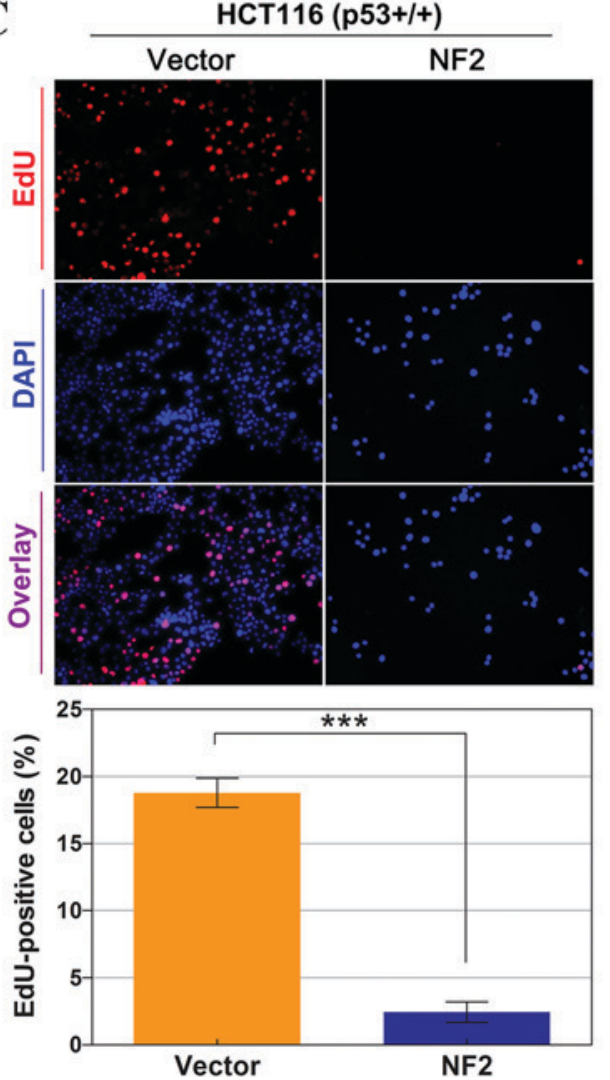

B

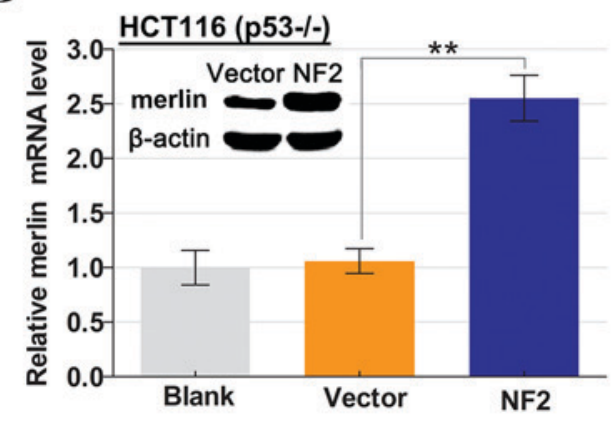

D
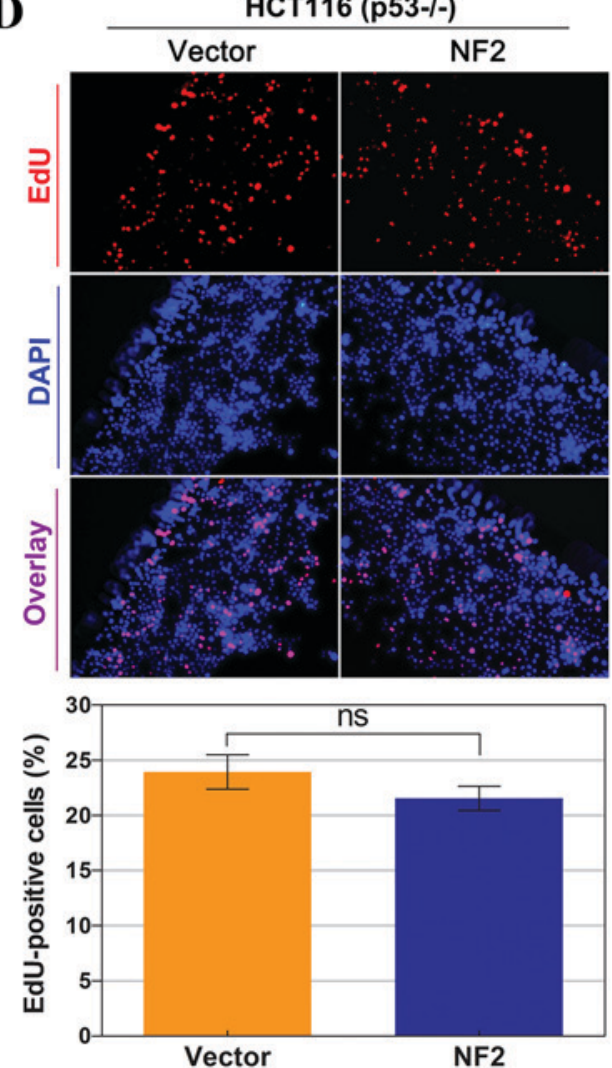

Figure 1.Effect of transient transfection-mediated merlin overexpression on the proliferation of HCT116 cell lines. Expression of merlin was measured by reverse transcription-polymerase chain reaction and western blotting, following treatment of (A) HCT116 p53 $3^{\mathrm{wt}}$ cultures and (B) p53/- cultures with $N F 2$-expressing vector or empty vector as a control. Representative images of EdU labelling and DAPI staining of (C) HCT116 p53 $3^{\mathrm{wt}}$ cultures and (D) p53/ cultures were captured under the fluorescence microscope at a magnification of x200. Quantification of EdU incorporating-cells was calculated as follows: EdU-positive cell numbers (red dots)/total numbers (blue dots) x100\%. At least three random images were taken from each well and counted by three individuals. Experiments were repeated 3 times. The errors bars represented standard deviation derived from triplicate experiments. ${ }^{* *} \mathrm{P}<0.01$ and ${ }^{* * * *} \mathrm{P}<0.001$ compared with controls.

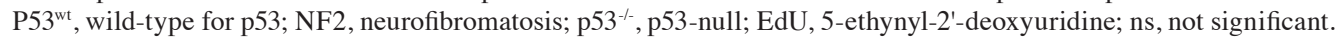

significant changes in protein levels of any of these cell-cycle regulators were observed in $\mathrm{HCT} 116 \mathrm{p} 53^{-/-}$cells upon merlin knockdown, which is in line with the results from the cell cycle analysis. In summary, the present results indicate that merlin loss may induce the downregulation of p53, and thus the dysregulation of cell-cycle regulatory proteins, including p21, cyclinD1/CDK4 and cyclin E1/CDK2 complexes. These cell-cycle regulatory proteins may mediate p53-dependent G1/S cell cycle progression (Fig. 5), and thereby perform a role in the initiation or development of merlin-deficient tumors.

\section{Discussion}

In a literature review study (28), merlin was reported to interact with numerous signaling components involved in cellular proliferation and cell cycle distributions. Recently, it was demonstrated that other pathways are associated with merlin, including p53/p21 signaling (19-21). There are similarities between merlin and $\mathrm{p} 53$, such as that merlin functions as a general tumor suppressor product to multiple cell types as p53 does, and notably, their tumor suppressive functions are associated with cell cycle control (25-27). The major difference 


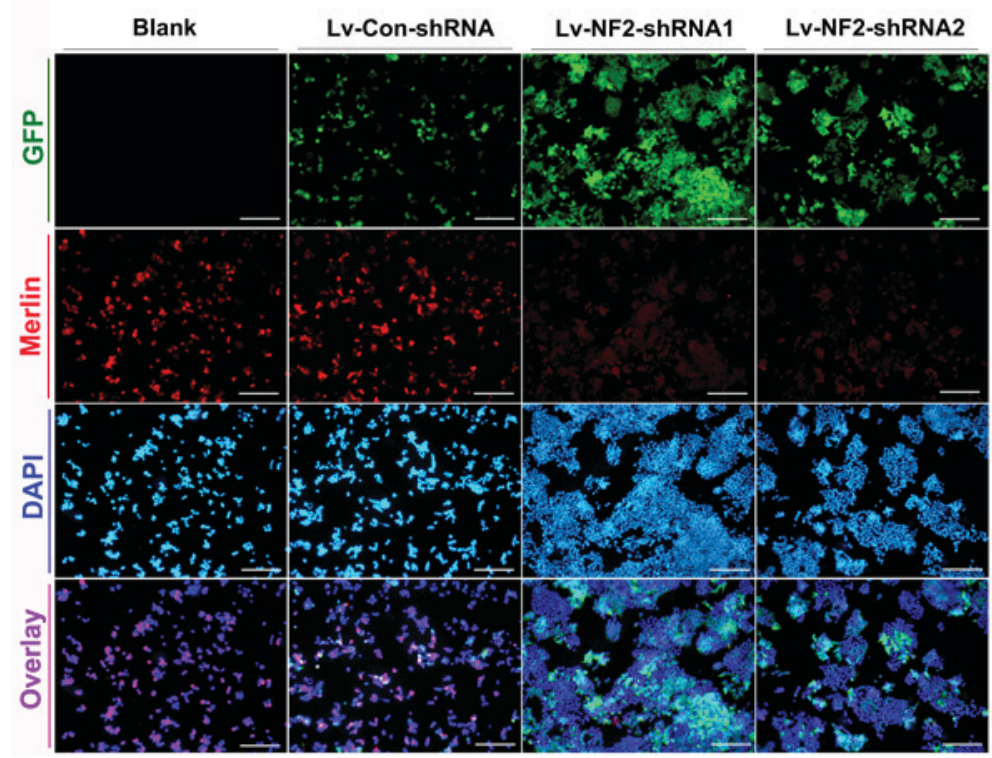

Figure 2. Lentivirus-mediated shRNAs targeting $N F 2$ in the HCT116 $\mathrm{p} 53^{\mathrm{wt}}$ cell line. Immunofluorescence was performed to verify merlin knockdown by comparing NF2 shRNA-transfected cultures (Lv-NF2-shRNAs) with non-transfected (Blank) or nonsense shRNA-transfected cultures (Lv-Con-shRNA). The percentage of GFP-positive (green) cells in total cell numbers (blue dots, DAPI staining) was evaluated under the fluorescence microscope to screen the efficiency of viral transfection. Scale bar=100 $\mu \mathrm{m}$. shRNA, short hairpin RNA; NF2, neurofibromatosis type 2; p53 ${ }^{\mathrm{wt}}$, wild-type for p53; Lv, lentivirus; GFP, green fluorescent protein; Con, control.

A

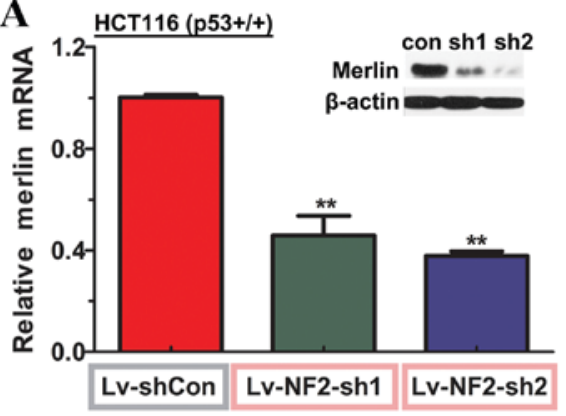

C

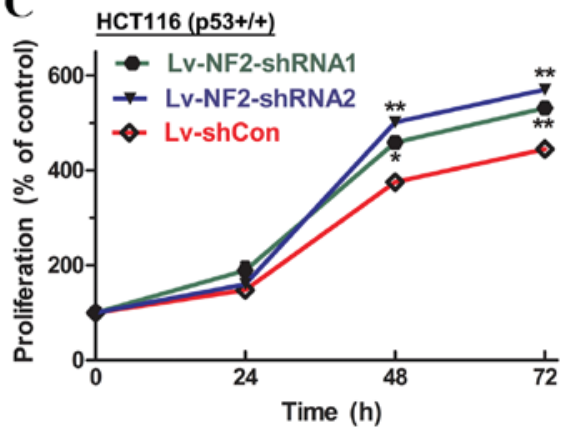

Lv-shCon Lv-NF2-sh1 Lv-NF2-sh2

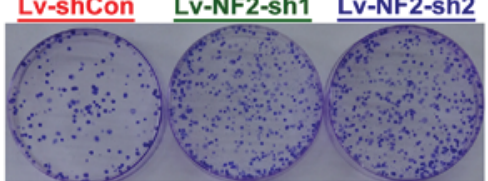

B

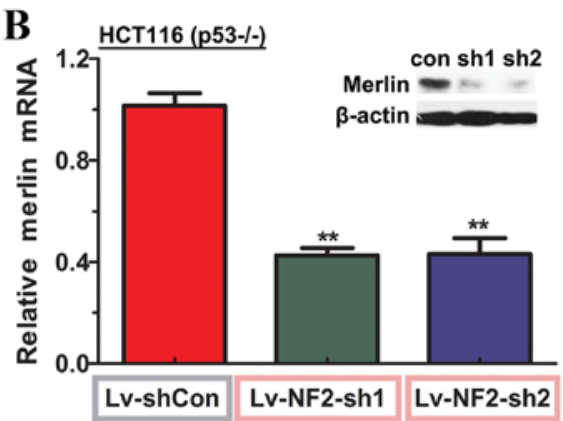

D
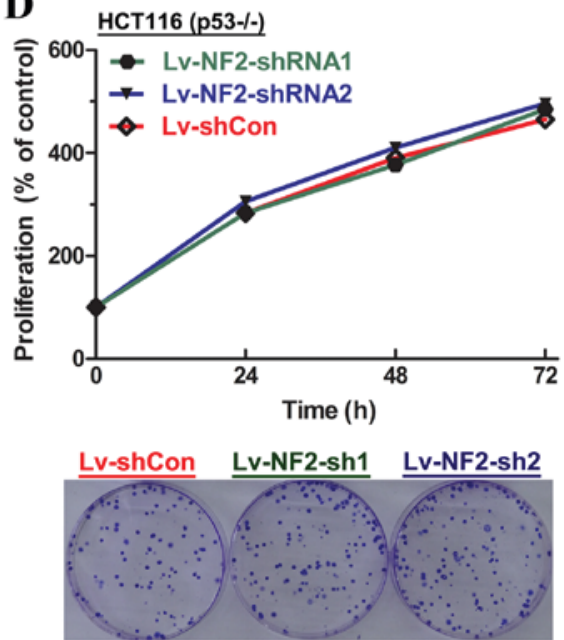

Figure 3. Effect of lentiviral shRNA-mediated merlin knockdown on the proliferation of HCT116 cell lines. At $72 \mathrm{~h}$ after transfection of lentiviral shRNAs, indicated HCT116 cell lines in experiment groups (sh1 and sh2) and control groups (Con) were harvested, divided and subjected to additional investigations. Knockdown efficiencies of NF2 shRNAs were assessed by reverse transcription-polymerase chain reaction and western blotting in (A) $\mathrm{p} 53^{+/+}$and (B) $\mathrm{p} 53^{-/}$with $\beta$-actin being used as a control. Cellular proliferation was determined by a Cell Counting Kit- 8 assay in (C) $\mathrm{p} 53^{+/+}$and (D) $\mathrm{p} 53^{-/}$. Numbers of colonies were then evaluated 7 days after seeding. Experiments (reverse transcription-polymerase chain reaction, western blotting and Cell Counting kit- 8 assay) were repeated 3 times with similar results. For colony formation assay, the experiment was performed 2 times with similar results. Con: Lv-Con-shRNA (Lv-shCon); sh: Lv-NF2-shRNA. " $\mathrm{P}<0.05$ and ${ }^{* *} \mathrm{P}<0.01$ compared with controls. sh, short hairpin; Con, control; NF2, neurofibromatosis type 2; p53 ${ }^{+/+} ; \mathrm{p} 53^{-/}$, p53-null; Lv, lentivrus. 
A

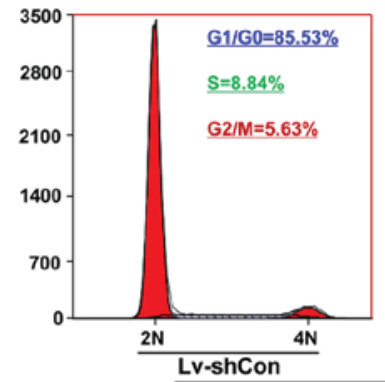

B

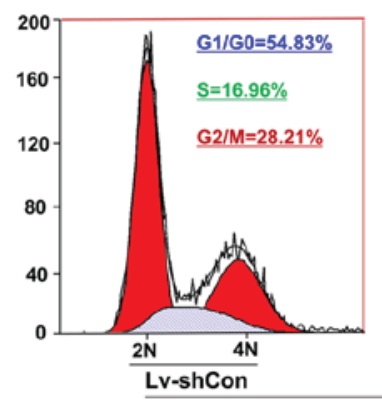

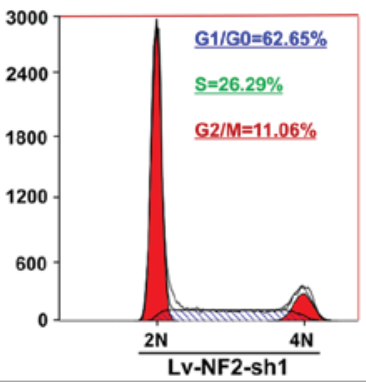

HCT116 (p53+/+)
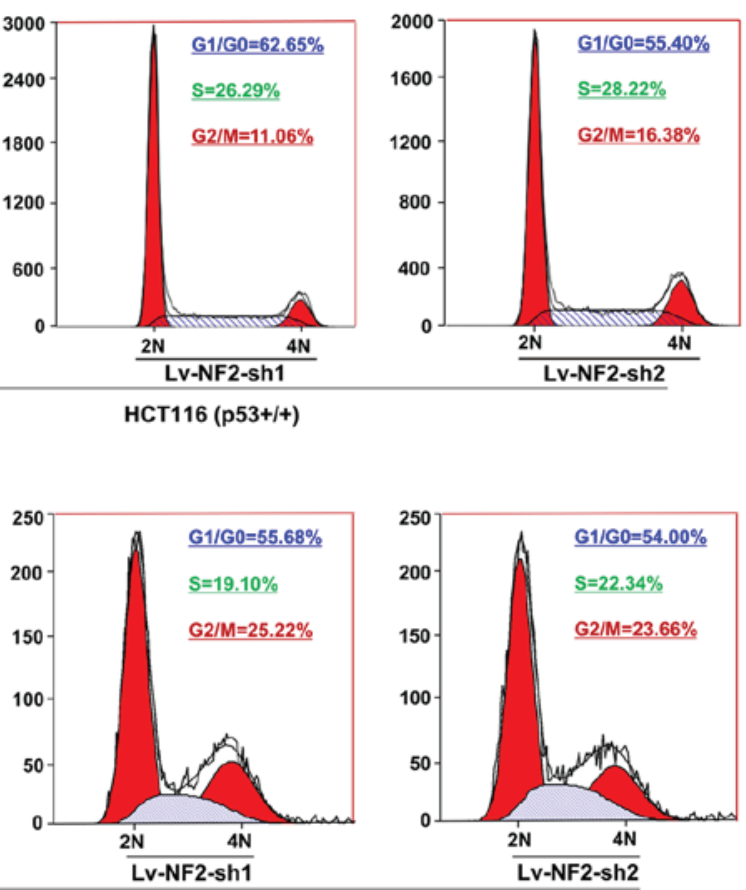

HCT116 (p53-I-)
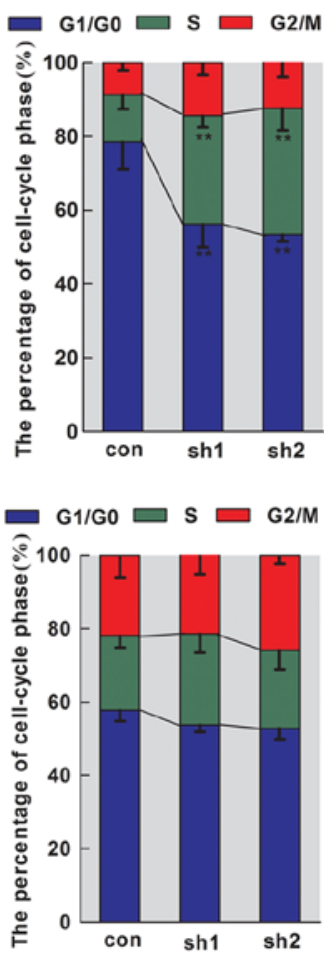

Figure 4. Alteration in the cell cycle pattern of HCT116 cell lines following transfection of merlin-targeted lentiviral shRNAs. Two cell types, (A) HCT116

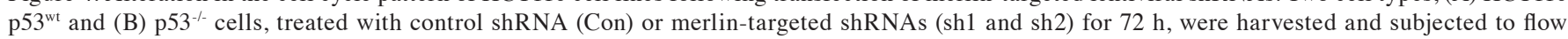
cytometric analysis of DNA content subsequent to propidium idodide staining. Representative micrographs and quantification of proportions of cells in specific cell-cycle phase (G0/G1, S or G2/M) were obtained from three independent experiments. Merlin knockdown rendered significantly reduced percentages of cells in the G1 phase (between $78.5 \pm 7.3$ and $56.3 \pm 6.3$ and $53.4 \pm 1.9 \%$ for sh1 and sh2, respectively) and increased percentages of cells in the S phase (between $12.8 \pm 3.9$ and $29.4 \pm 3.2$ and $34.2 \pm 5.9 \%$ for sh1 and sh2, respectively) in HCT116 p53 ${ }^{\text {wt }}$ cells. Two similar results from three independent experiments were used to plot a histogram with deviation bars. ${ }^{* *} \mathrm{P}<0.01$ compared with controls. sh, short hairpin; P53 ${ }^{\mathrm{wt}}$, wild-type for p53; NF2, neurofibromatosis; $\mathrm{p} 53^{-/}$, p53-null; Con, control.
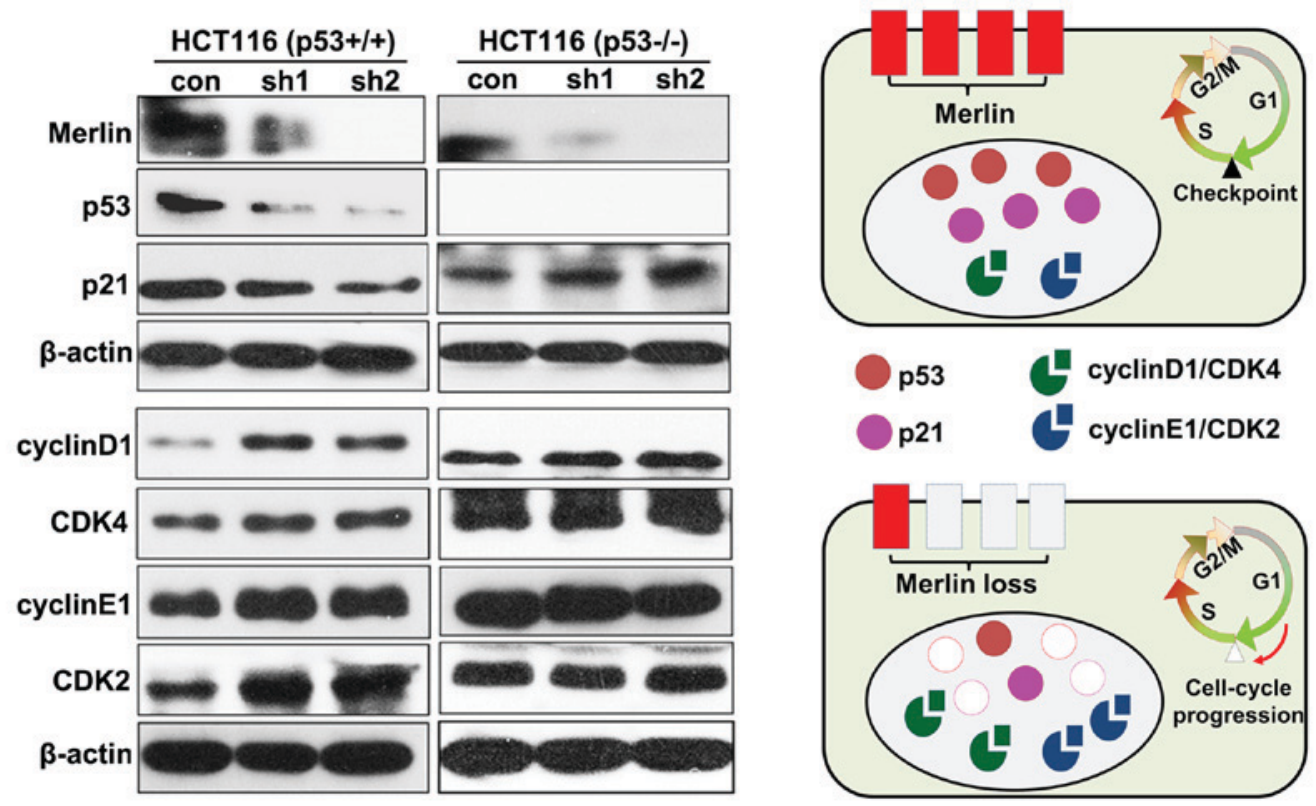

Figure 5. Role of p53-mediated pathways in cell-cycle progression driven by merlin knockdown. Expression of molecules involved in G1/S transition of the cell cycle in indicated HCT116 cell lines in the each of experiment groups and control groups was measured by western blotting. $\beta$-actin was used as the control. Proteins examined included merlin, p53, p21, cyclins D1/CDK4 and cyclin E1/CDK2 complexes. Schematic diagrams depicting the mechanisms of how merlin knockdown contributes to cell cycle progression are presented. p53 performs an important role in controlling cell-cycle progression by regulating the G1/S checkpoint in merlin-abundant cells; however, in the absence of merlin, p53 expression is inhibited followed by the dysregulation of the aforementioned cell-cycle regulatory proteins, thus promoting p53-dependent G1/S cell cycle progression. CDK, cyclin-dependent kinase; sh, short hairpin; con, control. 
is that they are predicted to localize into different subcellular compartments $(29,30)$. Merlin is localized preferentially to areas of membrane ruffles (29), whereas p53 is a nuclear phosphoprotein that functions as a sequence-specific transcription factor. Certain oncogenic activities require signaling from the cell membrane to the nucleus to initiate the cell's entrance into the cell cycle (30), raising the possibility that p53 may be one of the crucial mediators throughout the signal transmission path modulated by merlin. This theory was supported by the results from overexpression experiments, in which a significant inhibitory effect of overexpressed merlin on cellular proliferation was observed only in HCT116 $\mathrm{p} 53^{\mathrm{wt}}$ cells.

Merlin depletion in human/mouse normal Schwann cells was demonstrated to increase cellular proliferation, and therefore promote the tumorigenesis of schwannomas (31). In colorectal cancer, merlin deficiencies due to NF2 mutations tend to result in malignant progression (7). Notably, the HCT116 cell lines used in the present study had no detectable mutations in the NF2 gene through standard sequencing (data not shown), which is why RNA interference was performed to knock down merlin expression in merlin-abundant HCT116 cell lines in order to mimic tumor progression in vitro. The present study reported that knockdown of endogenous merlin resulted in enhanced cellular proliferation in the $\mathrm{p} 53^{\mathrm{wt}}$ cells but not in $\mathrm{p} 53^{-/-}$cells, indicating that merlin depletion may trigger uncontrolled cellular proliferation, one of the characteristics of malignancies $(6,7)$, at least in part through p53-dependent mechanisms.

Potential alterations in cell cycle patterns were also investigated, which may provide increased understanding into the effects of merlin knockdown on the cellular proliferation of HCT116 cell lines. For HCT116 cells with wild-type p53, merlin knockdown led to a significant decrease in the percentage of cells in the G0/G1 phase, which was accompanied by increased intra-S status. However, no significant changes for cell cycle distributions were observed in HCT116 cells lacking p53, indicating that loss of merlin tended to abrogate p53-involved G0/G1 arrest, and thereby promote G1- to S-phase progression.

It is well-known that p53 is induced in response to DNA damage, resulting in upregulation of $\mathrm{p} 21$, a potent cyclin-dependent kinase inhibitor that targets cyclin D1/CDK4,6 and cyclin E/CDK 2 complexes, thereby halting cell cycle progression either prior to S-phase entry or during the S-phase (19-21). Our previous study (21) reported that merlin loss was accompanied by repressed p21 expression and elevated levels of cyclin D1, CDK4, cyclin E1 and CDK2 proteins in schwannomas, in which p53 mutations are rarely reported (32). Accordingly, the present study demonstrated the presence of dysregulation of these cell-cycle regulators (p21 and cyclins/CDKs) only in the HCT116 $\mathrm{p}^{{ }^{\mathrm{wt}}}$ cell line upon knockdown of merlin, indicating that loss of merlin may trigger deregulated expression of G1/S transition genes mainly through a p53-dependent manner. Thus, the present study provides additional information regarding the causes and detailed events of merlin-deficient cell cycle progression.

In conclusion, to the best of our knowledge, the present study reported for the first time that loss of merlin contributes to cellular proliferation and cell cycle progression through p53-dependent mechanisms. p53 is a molecule that should be investigated for its potential in targeted drug therapy for merlin-deficient malignancies, although verifications remain to be performed in more cell types whose tumorigenicity is associated with $N F 2 /$ merlin.

\section{Acknowledgements}

The present study was funded by the National Natural Science Foundation of China (grant nos. 81371086 and 81670919 to Zhaoyan Wang and no. 81570906 to Hao Wu).

\section{References}

1. Wang Z, Lu Y, Tang J, Wang H and Wu H: The phosphorylation status of merlin in sporadic vestibular Schwannomas. Mol Cell Biochem 324: 201-206, 2009.

2. Zhang Z, Wang Z, Sun L, Li X, Huang Q, Yang T and Wu H: Mutation spectrum and differential gene expression in cystic and solid vestibular schwannoma. Genet Med 16: 264-270, 2014.

3. Chen H, Zhang X, Zhang Z, Yang T, Wang Z and Wu H: The role of NF2 gene mutations and pathogenesis-related proteins in sporadic vestibular schwannomas in young individuals. Mol Cell Biochem 392: 145-152, 2014.

4. Lau YK, Murray LB, Houshmandi SS, Xu Y, Gutmann DH and Yu Q: Merlin is a potent inhibitor of glioma growth. Cancer Res 68: 5733-5742, 2008.

5. Sheikh HA, Tometsko M, Niehouse L, Aldeeb D, Swalsky P, Finkelstein S, Barnes EL and Hunt JL: Molecular genotyping of medullary thyroid carcinoma can predict tumor recurrence. Am J Surg Pathol 28: 101-106, 2004.

6. Sekido Y: Genomic abnormalities and signal transduction dysregulation in malignant mesothelioma cells. Cancer Science 101: 1-6, 2010.

7. Cačev T, Aralica G, Lončar B and Kapitanović S: Loss of NF2/Merlin expression in advanced sporadic colorectal cancer. Cell Oncol (Dordr) 37: 69-77, 2014.

8. Shimizu K, Nagamachi Y, Tani M, Kimura K, Shiroishi T, Wakana S and Yokota J: Molecular cloning of a novel NF2/ERM/4.1 superfamily gene, ehm2, that is expressed in high-metastatic K1735 murine melanoma cells. Genomics 65 : $113-120,2000$

9. Sainz J, Huynh DP, Figueroa K, Ragge NK, Baser ME and Pulst SM: Mutations of the neurofibromatosis type 2 gene and lack of the gene product in vestibular schwannomas. Hum Mol Genet 3: 885-891, 1994.

10. Stamenkovic I and Yu Q: Merlin, a 'magic' linker between the extracellular cues and intracelular signaling pathways that regulate cell motility, proliferation, and survival. Curr Protein Pept Sci 11: 471-484, 2010.

11. Schroeder RD, Angelo LS and Kurzrock R: NF2/merlin in hereditary neurofibromatosis 2 versus cancer: Biologic mechanisms and clinical associations. Oncotarget 5: 67-77, 2014.

12. Gutmann DH, Giordano MJ, Fishback AS and Guha A: Loss of merlin expression in sporadic meningiomas, ependymomas and schwannomas. Neurology 49: 267-270, 1997.

13. Yang C, Asthagiri AR, Iyer RR, Lu J, Xu DS, Ksendzovsky A, Brady RO, Zhuang Z and Lonser RR: Missense mutations in the NF2 gene result in the quantitative loss of merlin protein and minimally affect protein intrinsic function. Proc Natl Acad Sci USA 108: 4980-4985, 2011.

14. Ammoun S, Flaiz C, Ristic N, Schuldt J and Hanemann CO: Dissecting and targeting the growth factor-dependent and growth factor-independent extracellular signal-regulated kinase pathway in human schwannoma. Cancer Res 68: 5236-5245, 2008.

15. Curto M, Cole BK, Lallemand D, Liu CH and McClatchey AI: Contact-dependent inhibition of EGFR signaling by Nf2/Merlin. J Cell Biol 177: 893-903, 2007.

16. Flaiz C, Ammoun S, Biebl A and Hanemann CO: Altered adhesive structures and their relation to RhoGTPase activation in merlin deficient Schwannoma. Brain Pathol 19: 27-38, 2009.

17. Li W, You L, Cooper J, Schiavon G, Pepe-Caprio A, Zhou L, Ishii R, Giovannini M, Hanemann CO, Long SB, et al: Merlin/NF2 suppresses tumorigenesis by inhibiting the E3 ubiquitin ligase CRL4(DCAF1) in the nucleus. Cell 140: 477-490, 2010 
18. Robanus-Maandag E, Giovannini M, van der Valk $M$, Niwa-Kawakita M, Abramowski V, Antonescu C, Thomas G and Berns A: Synergy of Nf2 and p53 mutations in development of malignant tumours of neural crest origin. Oncogene 23: 6541-6547, 2004.

19. Chang Z, Guo CL, Ahronowitz I, Stemmer-Rachamimov AO, MacCollin M and Nunes FP: A role for the p53 pathway in the pathology of meningiomas with NF2 loss. J Neurooncol 91: 265-270, 2009.

20. Kim H, Kwak NJ, Lee JY, Choi BH, Lim Y, Ko YJ, Kim YH, Huh PW, Lee KH, Rha HK and Wang YP: Merlin neutralizes the inhibitory effect of Mdm2 on p53. J Biol Chem 279: 7812-7818, 2004.

21. Wu H, Chen Y, Wang ZY, Li W, Li JQ, Zhang L and Lu YJ: Involvement of p21 (waf1) in merlin deficient sporadic vestibular schwannomas. Neuroscience 170: 149-155, 2010

22. Livak KJ and Schmittgen TD: Analysis of relative gene expression data using real-time quantitative PCR and the 2(-Delta Delta C(T)) Method. Methods 25: 402-408, 2001

23. Poulikakos PI, Xiao GH, Gallagher R, Jablonski S, Jhanwar SC and Testa JR: Re-expression of the tumor suppressor NF2/merlin inhibits invasiveness in mesothelioma cells and negatively regulates FAK. Oncogene 25: 5960-5968, 2006.

24. Schulze KM, Hanemann CO, Müller HW and Hanenberg $\mathrm{H}$ : Transduction of wild-type merlin into human schwannoma cells decreases schwannoma cell growth and induces apoptosis. Hum Mol Genet 11: 69-76, 2002.

25. Lü J, Zou J, Wu H and Cai L: Compensative shuttling of merlin to phosphorylation on serine 518 in vestibular schwannoma. Laryngoscope 118: 169-174, 2008.
26. Muranen T, Grönholm M, Renkema GH and Carpén O: Cell cycle-dependent nucleocytoplasmic shuttling of the neurofibromatosis 2 tumour suppressor merlin. Oncogene 24: 1150-1158, 2005.

27. Arellano M and Moreno S: Regulation of CDK/cyclin complexes during the cell cycle. Int J Biochem Cell Biol 29: 559-573, 1997.

28. Scoles DR: The merlin interacting proteins reveal multiple targets for NF2 therapy. Biochim Biophys Acta 1785: 32-54, 2008.

29. Lallemand D, Manent J, Couvelard A, Watilliaux A, Siena M, Chareyre F, Lampin A, Niwa-Kawakita M, Kalamarides M and Giovannini M: Merlin regulates transmembrane receptor accumulation and signaling at the plasma membrane in primary mouse Schwann cells and in human schwannomas. Oncogene 28: 854-865, 2009.

30. Planque N: Nuclear trafficking of secreted factors and cell-surface receptors: New pathways to regulate cell proliferation and differentiation and involvement in cancers. Cell Commun Signal 4: 7, 2006.

31. Ahmad Z, Brown CM, Patel AK, Ryan AF, Ongkeko R and Doherty JK: Merlin knockdown in human Schwann cells: Clues to vestibular schwannoma tumorigenesis. Otol Neurotol 31: 460-466, 2010.

32. Monoh K, Ishikawa K, Yasui N, Mineura K, Andoh H and Togawa K: p53 tumor suppressor gene in acoustic neuromas. Acta Otolaryngol Suppl 537: 11-15, 1998. 